\title{
A survey of selected animal-based measures of dairy cattle welfare in the Eastern Alps: Toward context-based thresholds
}

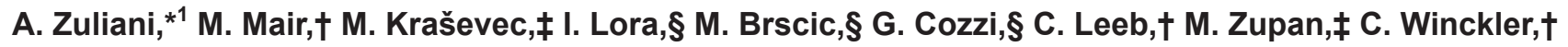 \\ and S. Bovolenta* \\ *Department of Food, Agricultural, Environmental and Animal Sciences, University of Udine, 33100 Udine, Italy \\ †Department of Sustainable Agricultural Systems, University of Natural Resources and Life Sciences, 1180 Vienna, Austria \\ ‡Department of Animal Science, University of Ljubljana, 1230 Domžale, Slovenia \\ $\S$ Department of Animal Medicine, Production and Health, University of Padova, 35020 Legnaro, Italy
}

\begin{abstract}
In the Alps, traditional dairy farms are small-scale operations where vertical transhumance from valley indoor housing systems to highland pasture-based systems is still practiced in summer. Vertical transhumance implies a substantial change of environment, available resources, and management practices from one season to another. In such systems, animal-based welfare measures need to be monitored throughout the year to capture the variation of welfare outcomes, based on which targeted welfare management plans can be implemented. Because the Welfare Quality assessment approach has been tailored to indoor housing and intensive farming systems, the European Food Safety Authority recently developed a welfare assessment protocol for small-scale dairy cattle farms adapted after the Welfare Quality framework. The aim of this study was to assess nonbehavioral animal-based measures as defined by this protocol at different time points for transhumant systems in the Alps. In total, 18 animal-based measures were assessed before, during, and after the mountain pasture period in a sample of 67 small-scale dairy cattle farms practicing vertical transhumance in neighboring provinces of Austria, Italy, and Slovenia. Significant differences between assessments were identified for dirtiness of legs and teats, hairless patches, lesions and swellings, claw condition, ocular discharge, and diarrhea whereas BCS, lameness/severe lameness, vulvar discharge, nasal discharge, and hampered respiration were unchanged between seasons. In addition, a benchmarking exercise was carried out to identify relative boundaries (worst quartile thresholds) for each animal-based measure and to contribute to the discussion about achievable welfare outcomes for the 2 husbandry conditions that charac-
\end{abstract}

Received May 30, 2017.

Accepted October 6, 2017.

${ }^{1}$ Corresponding author: zuliani.anna.2@spes.uniud.it terize a transhumant system. Worst quartile thresholds indicated a high prevalence of dirtiness $(>80 \%)$ when cows were kept indoors, high prevalence of hairless patches $(65 \%)$ before pasture turnout, and high prevalence of very lean cows $(>13 \%)$ throughout the assessments. On the other hand, the best quartile thresholds for most clinical conditions suggested that high welfare standards (zero prevalence) are widely achievable in mountain farms practicing vertical transhumance during all assessments. The thresholds identified through benchmarking should serve as the basis for an effective context-based welfare management strategy promoting continuous welfare improvement on-farm.

Key words: mountain farm, small-scale, animal welfare, benchmarking

\section{INTRODUCTION}

Animal welfare has become a major public concern in the debate regarding sustainable livestock farming (Fraser, 2005), and societal expectations toward improved animal welfare have to be met by all farming systems. To ensure animal welfare, robust assessment methods are required. In Europe, the Welfare Quality project (Blokhuis, 2008) has been one of the most important efforts toward the development of on-farm welfare assessment systems aiming at transferring science-based measures into reliable and transparent information for consumers. However, the framework and the measures therein are tailored to indoor housing and intensive farming systems (Welfare Quality, 2009). Despite the ongoing global agricultural transition to intensive and large-scale farming systems, small-scale farms are still the majority in Europe. Mountain farms are one example of traditional small-scale operations (Wymann von Dach et al., 2013), characterized by their environmental and topographical conditions. To address the lack of information regarding welfare assessment in small-scale dairy farms, the European Food Safety Authority (EFSA) provided a scientific opinion 
on the feasibility of current welfare assessment methods in so-called nonconventional small-scale dairy farming settings (characterized by, for example, $\leq 75$ lactating cows, dual-purpose or local breeds, family-run farms). One of the outcomes was a protocol modified after the Welfare Quality protocol (WQ) applicable to smallscale farming systems (EFSA, 2015) in which cows have access to pasture year round, seasonally (summer), or no access to pasture at all. This protocol was tested on 124 farms in 4 European countries (Austria, France, Italy, and Spain). However, for reasons of consistency, the assessments were performed during winter; thus, cows in mountain farms with summer grazing on highland pastures were only evaluated indoors.

Recently, the importance of performing dairy cattle welfare assessment using animal based-measures $(\mathbf{A B M})$ and acknowledging context-based variability in welfare outcomes was emphasized by the World Animal Health Organization (OIE, 2015) and the International Organization for Standardization (ISO, 2016). Animal based-measures are direct indicators of animal welfare and reflect, among other things, the context of each farming system (i.e., environment, resources, and management practices). For example, dairy cattle farming in the Alps is often characterized by indoor housing during cold months and a pasture-based system during summer. Moreover, in some regions, summer grazing takes place at a higher elevation than the areas where winter farming takes place (Battaglini et al., 2014). This vertical transhumance aims to exploit pastures located at higher altitudes during the warmest months of the year (i.e., from June to September). Most farmers also let cows graze on valley pastures before and after transhumance to facilitate the transition from the indoor management and feeding system to the highland pasture-based system. This management practice implies a substantial change of environment, available resources, and management practices. Thus, in transhumant systems combining indoor winter housing and pasture-based husbandry during summer, ABM would need to be monitored throughout the year to capture the variation of welfare outcomes resulting from the change in context and to address the main issues characterizing each context.

The aim of this study was to assess ABM, focusing on nonbehavioral clinical outcomes, at different time points in a sample of small-scale dairy cattle farms practicing vertical transhumance in the Eastern Alps (Italy, Austria, and Slovenia). The results could help to identify critical welfare conditions, add to the discussion around achievable welfare outcomes for the different husbandry conditions that characterize a transhumant system, and inform targeted welfare management strategies.

\section{MATERIALS AND METHODS}

\section{Study Area and Farms}

The survey was carried out in 67 small-scale dairy farms located in the Eastern Alps (Table 1). The study area is characterized by mountains and encompassed neighboring provinces of 3 European countries: Austria (Innsbruck, Tiroler Oberland, Tiroler Unterland; $\mathrm{n}=$ 25), Slovenia (Osrednjeslovenska, Gorenjska, Goriška; n $=21$ ), and Italy (Trento, Bolzano, Vicenza, Udine; $\mathrm{n}=$ 21). The farms involved were selected through breeders' associations, dairy farmers' organizations, and organic farming associations, as well as personal contacts, and all met the criteria to be considered small-scale farms according to the EFSA (2015) definition. Most farmers sold their products through cooperatives $(73 \%)$, which produced mainly according to either the European "Geographical Indication" or "organic" certification schemes (54\%). Moreover, only farmers practicing the traditional seasonal movement of the entire herd from valley farms to highland pastures during summer were included. Tie-stall systems were predominant (almost $70 \%$ ) in the sample, as expected for small-scale systems in general and mountain farming systems during winter specifically (Sturaro et al., 2013; Nash et al., 2016).

Table 1. Farm descriptors of 67 alpine dairy farms practicing vertical transhumance

\begin{tabular}{lrrrr}
\hline Farm descriptor & Mean & \multirow{2}{*}{ SE } & Minimum & Maximum \\
\hline Dairy cows (no.) & 22 & 1.7 & 6 & 63 \\
Milk yield (kg/cow per year) & 5,928 & 183 & 3,330 & 10,200 \\
Pasture (h/yr) & 3,080 & 167 & 1,200 & 11,568 \\
Concentrate at peak lactation (kg/cow per day) & 5 & 0.3 & 0 & 10 \\
Family members working in the farm (no.) & 3 & 0.1 & 1 & 6 \\
Income from dairy production (\%) & 67 & 3.4 & 10 & 100 \\
Farm surface (ha) & 18 & 1.6 & 0 & 80 \\
Altitude: winter farm (m above sea level) & 622 & 43.9 & 6 & 1,500 \\
Altitude: summer farm (m above sea level) & 1,392 & 37.5 & 710 & 2,096 \\
\hline
\end{tabular}




\section{ENVIRONMENT RESOURCES}

WELFARE

OUTCOMES

\begin{tabular}{|c|c|c|}
\hline Family size/education/skills & Forage \\
\hline Farm size/location & Concentrate & Dutritional state \\
\hline Species/breed type & Pasture access & Daseases \\
\hline Certifications/commercialization & Housing & Behavior \\
\hline Income/productivity/diversification & Treatments/procedures & Longevity/mortality \\
\hline
\end{tabular}

Figure 1. Framework used for data collection in small-scale dairy farms of the Eastern Alps. From left to right, the graph displays distal and proximal factors (environment and resources, respectively) affecting welfare outcomes in cows.

\section{Data Collection}

The assessment protocol for nonbehavioral ABM of welfare followed the methodology proposed by EFSA for small-scale dairy farms (EFSA, 2015). Although based on the WQ framework (Welfare Quality, 2009), the EFSA protocol differs from the latter in some measures. Records of coughing episodes were removed from the protocol because the EFSA working group considered evaluation of this measure too time consuming. Instead, 2 additional measures were included because they were considered relevant for small-scale systems: longevity (expressed as the percentage of cows in fourth lactation or higher) and claw condition (classified as good condition or overgrown). The measure addressing ocular discharge was redefined by adding a new category (i.e., distinguishing between serous and purulent ocular discharge). Similarly, teats were considered separately from the rest of the udder when scoring for soiling.

All ABM were collected complying with the EFSA adapted protocols. They were divided into ABM observed (ABMo) and ABM recorded from farm records (ABMr). The former included BCS, soiling, integument alterations (hairless patches, lesions, swellings, and claw overgrowth), and clinical conditions (lameness/severe lameness, ocular discharge, nasal discharge, vulvar discharge, hampered respiration, and diarrhea). The ABMr aimed at retrieving information on longev- ity, incidence of downer cows, dystocia, sudden deaths or emergency slaughter/euthanasia (i.e., "mortality"), and milk SCC from farm records during a 12-mo period. In addition, detailed information on farm characteristics, management, and resources were collected to shed light on the Eastern Alpine farming system and its practices (Figure 1). Assessments were carried out 3 times on each farm to capture the variation of nonbehavioral ABM that occur with the seasonal changes in the Eastern Alps. Farm visits were divided in 2 parts: first, animals and resources were assessed in the barn or on pasture, and second, the farmer was interviewed to retrieve data on management practices and farm records. Sample size was determined according to WQ guidelines, which requires assessing all animals in herds of 30 or less and up to 50 animals in larger farms (maximum herd size 63 animals).

The first assessment was performed on the home farm at least 2 wk before cows were moved to highland pasture (before pasture, BP); the second assessment was performed on pasture 5 to 7 wk after cows were moved to highland pasture (during pasture, DP); and the third assessment was performed on the home farm 2 to 4 wk after the cows had returned from the mountain pasture (after pasture, AP).

Assessors with a veterinarian or animal science background attended ad hoc training on the EFSA protocol using video clips, photographs, and direct examination of 20 cows. Agreement between observers and the 
trainer was tested using the kappa statistic. Observers not meeting the criterion of sufficient agreement $(\kappa>$ 0.6 ) were retrained through the provision of additional video clips and photographs.

\section{Statistical Analysis}

Summary statistics were performed for each set of animal-based measures obtained from observations $(\mathrm{ABMo})$ and from farm records $(\mathrm{ABMr})$. The effect of the time of assessment (BP, DP, and AP) on the prevalence of the different $\mathrm{ABM}$ o was tested using Friedman's test because no measure displayed a normal distribution. When an overall significant effect of the assessment was detected, post hoc pairwise comparisons were performed using the Wilcoxon test and with Bonferroni correction. Moreover, quartiles were identified for $\mathrm{ABM}$ and $\mathrm{ABMr}$ from all herd-level measures to suggest critical or achievable levels applicable to smallscale mountain dairy farms for the indoor housed and on pasture conditions (de Vries et al., 2013). Measures with zero prevalence were excluded from the benchmarking exercise.

\section{RESULTS AND DISCUSSION}

\section{Farm and Management Descriptors}

The 67 farms involved in the study (Table 1) were located, on average, $622 \mathrm{~m}$ above sea level (asl) during winter and 1,392 $\mathrm{m}$ asl during summer. The mean herd size was 22 lactating cows and Simmental was the most prevalent breed. The average milk yield was estimated to be just below $6,000 \mathrm{~kg} / \mathrm{cow}$ per year. In addition to highland pasture areas, each farm had, on average, 18 ha of land for forage production and valley grazing between seasons. Three family members were usually employed in dairy farming, which contributed to $70 \%$ of the family's income. Cows were allowed to graze and exercise on both valley and highland pasture for an overall mean amount of about 3,000 h/yr. The average amount of concentrate given to cows at peak lactation was estimated to be $5 \mathrm{~kg} / \mathrm{cow}$ per day.

Most farmers (64\%) practiced seasonal calving (i.e., winter calving) to match the lower production and thus lower energy requirements of the alpine pasture-based feeding system (Horn et al., 2013; Piccand et al., 2013). Functional foot trimming was practiced once a year in almost all farms, mostly before pasture turnout. A slight majority of farms (54\%) did not practice routine disbudding of calves and when practiced, no analgesics or anesthetics were used. Natural mating was not commonly practiced in the sample of farms. In terms of treatments, around $40 \%$ of farmers declared that they had treated at least one cow for mastitis during the last year, whereas only $18 \%$ of farmers declared that they used antiparasitics before or during pasture turnout.

\section{Animal-Based Measures}

A total of 3,702 cows were assessed in the course of the study. Summary statistics for ABMo and ABMr are given in Table 2 and Table 3, respectively. In terms of body condition, no significant differences in the prevalence of lean cows were observed between assessments (BP, DP, and AP), similar to findings of Corazzin et al. (2010) and Peric et al. (2017) in transhumant systems in the Eastern Alps. In contrast, Burow et al. (2013) reported an increased prevalence of very lean cows when animals were moved from winter indoor housing to summer pasture in Denmark. This difference might be linked to the high-yielding breeds (i.e., Holstein) and the lack of seasonal-calving practices used in Danish dairy systems, resulting in a decrease in BCS when cows are moved to pasture during early lactation stages (Roche et al., 2007; Piccand et al., 2013) in addition to the natural variability of forage quality due to seasonal and altitudinal differences.

The percentage of animals with clean legs and teats was significantly higher in DP than BP, but cleanliness deteriorated again in AP. The positive, but short, effect of pasture on cleanliness observed here might be driven by the difference in housing systems and thus availability of clean lying areas between assessments (i.e., winter tie-stall system and summer free-range pasture-based system). However, although the median percentage of cows with dirty legs when indoors was higher than reported by Whay et al. (2003) for loose-housing systems and Popescu et al. (2013) for tie-stall systems, the median rates of dirty teats (minor splashing included) observed throughout the study were lower (below 14\%). This might be due to the greater attention paid to teat cleanliness in small herds compared with larger herds (i.e., where less time is available for cleaning routine) as well as to the time at which farms were visited (i.e., soon after milking).

The prevalence of integument alterations (i.e., lesions, swellings, and hairless patches) on both legs and body was significantly lower in AP compared with $\mathrm{BP}$ and DP. Additionally, in indoor systems (BP and AP), the median percentage of cows per farm with integument alterations was lower than what has been reported in several studies throughout Europe (e.g., Whay et al., 2003; Mattiello et al., 2011; Popescu et al., 2013).A beneficial effect of pasture on integument alterations, due to low friction and the absence of constraining envi- 
Table 2. Prevalence of animal-based measures observed (ABMo) in 67 alpine dairy farms before $(\mathrm{BP}, \mathrm{n}=1,206)$, during $(\mathrm{DP}, \mathrm{n}=1,197)$, and after pasture season (AP, $\mathrm{n}=1,299)$

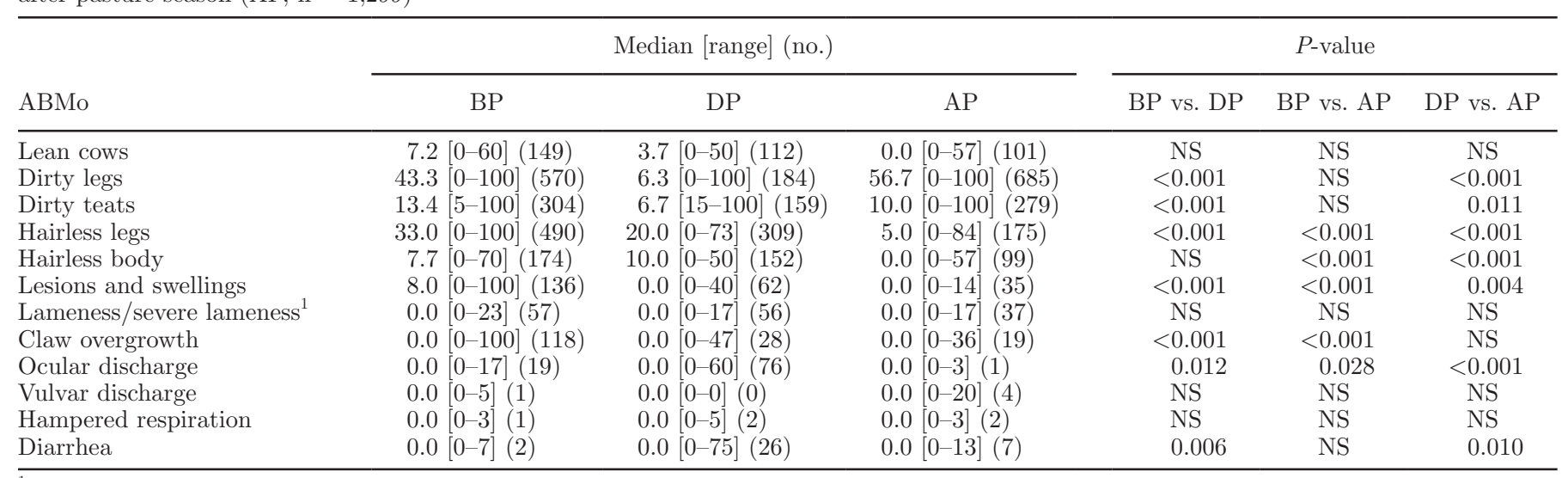

${ }^{1}$ Cows classified as lame in tie-stall systems or severely lame in loose-housing systems.

ronments where collisions are more likely, was observed in many other studies carried out in both mountain and lowland areas (Keil et al., 2006; Corazzin et al., 2010; Burow et al., 2013).

The median prevalence of lameness or severe lameness (tie-stalls or loose housing, respectively) was $0 \%$ for all assessments, and no effect of the change in husbandry system was found. However, this result has to be interpreted cautiously. The lameness scoring system used for tie-stall systems distinguished between nonlame and lame animals only and was less sensitive in detecting mild lameness compared with the 3-step locomotion scoring method used in loose-housing systems, which differentiates between mildly and severely lame animals (Leach, et al., 2009). Therefore, overall lameness prevalence is presented for tie-stall systems (thus including also a part of the mild lameness cases), whereas only severe lameness prevalence is reported for loose-housing systems. The unexpected lack of an effect of the assessment may, on the one hand, be due to the focus on the usually less prevalent severe lameness but it may also be explained by the higher attention paid by farmers to claw health in transhumant systems (Regula et al., 2004), to the less demanding yields characterizing small-scale systems, resulting in fewer productivity-related clinical conditions (Oltenacu and Broom, 2010), or to the beneficial effect of farming systems with seasonal pasture access (Rutherford et al., 2009).

No cases of nasal discharge were observed and, in accordance with several studies carried out in indoor systems throughout Europe (e.g., Coignard et al., 2013; de Vries et al., 2013; Popescu et al., 2013; Zuliani et al., 2017), low prevalences for the other clinical measures (i.e., ocular discharge, vulvar discharge, hampered respiration, and diarrhea) were found throughout the assessments. Nevertheless, both ocular discharge and diarrhea were significantly more prevalent in DP than in BP and subsequently decreased again in AP. For ocular discharge, prevalence in the AP period was also lower than in the BP period. The increased prevalence of liquid feces in cows on pasture may reflect the diet change from dry to mainly fresh forage (Corazzin et al., 2010; Burow et al., 2013). The findings for ocular discharge might be related to the presence of flies and their feeding behavior on cattle eye secretions, especially when on pasture (Palacio et al., 2015).

Concurrent with low prevalences of clinical findings throughout this study, the AMBr (Table 3) showed a low prevalence. For example, the low median rates of dirty

Table 3. Summary statistics for animal-based measures retrieved from farm records (ABMr) in 67 alpine dairy farms and related expert-based critical welfare (alarm) thresholds defined by Welfare Quality (2009)

\begin{tabular}{|c|c|c|c|c|c|c|}
\hline $\begin{array}{l}\mathrm{ABMr} \\
(\% \text { of cows during last } 12 \mathrm{mo})\end{array}$ & Minimum & 25 th & Median & 75th & Maximum & Alarm threshold \\
\hline Fourth lactation or higher & 0.0 & 26.3 & 35.3 & 50.0 & 69.2 & $\mathrm{NA}^{1}$ \\
\hline Dystocia & 0.0 & 0.0 & 0.0 & 2.5 & 14.3 & 5.5 \\
\hline $\mathrm{SCC}>400,000$ cells $/ \mathrm{mL}$ & 0.0 & 0.0 & 5.7 & 15.0 & 78.8 & 17.5 \\
\hline Mortality & 0.0 & 0.0 & 0.0 & 2.1 & 23.1 & 4.5 \\
\hline
\end{tabular}

${ }^{1} \mathrm{NA}=$ no reference available. 
teats might have had a positive effect on SCC (median of $5.7 \%$ of cows/farm per year had SCC >400,000 cells/ $\mathrm{mL}$ ), similar to that described by Zuliani and colleagues (2017) in traditional mountain farms. In addition, low prevalence of clinical findings such as lameness/severe lameness might have contributed to longevity and productive lifetime (i.e., median of $35 \%$ of cows per farm were at their fourth lactation or higher).

\section{Context-Based Thresholds}

Assessment protocols adopting ABM have the potential to provide a common currency for animal welfare status across a diversity of husbandry systems. However, 2 main approaches have been followed to identify critical thresholds for ABM depending on the purpose of the assessment. One approach is to ask experts for their opinion regarding unacceptable/acceptable thresholds above/below which welfare would be compromised. Experts have also been asked to rank ABM according to their relative importance (de Graaf et al., 2017). This approach aims to identify acceptable welfare conditions in terms of effect on the animal; it was applied by the WQ Consortium (WQ, 2009) among others to assessment protocols for indoor systems and, later, for pasture-based systems (Burow et al., 2013).

In recent years, a second approach has emerged that uses benchmarking of on-farm data as a way to induce change by identifying critical thresholds at which action is needed based on ABM collected in a sample of farms. The best- and worst-performing groups are defined by data distribution (e.g., quartiles), and welfare improvement is pursued by targeting achievable welfare outcomes as obtained by peers. Despite having different aims, a benchmarking approach was applied by von Keyserlingk et al. (2012) in North America and by de Vries et al. (2013) in Europe as well as within private initiatives (e.g., Business Benchmark on Farm Animal Welfare; https://www.bbfaw.com).

Because the establishment of thresholds for ABM in welfare management plans is also embedded in the recent ISO technical specification on animal welfare (ISO, 2016), it is important to understand how thresholds would work in different husbandry systems, such as those characterizing the transhumant system of the Eastern Alps. Figure 2 displays thresholds for ABM for the BP, DP, and AP assessments as derived from a benchmarking approach (i.e., best and worst quartiles) in our sample of small-scale mountain farms. It is interesting to note that thresholds for some ABM differ according to the time of assessment and are often quite distinct from both expert-opinion thresholds and worst quartile thresholds proposed in other studies. For example, the worst quartile threshold in this study for very lean cows is a prevalence of 26,20 , and $14 \%$ for $\mathrm{BP}, \mathrm{DP}$, and AP, respectively. These prevalences would translate to "Absence of prolonged hunger" scores of approximately 20,30 , and 50, respectively, according to the expert opinions gathered in the course of the WQ project (Welfare Quality, 2009). The WQ scores range from 0 to 100 (with 100 indicating the best condition); thus, both BP and DP thresholds would be considered by the WQ experts to be not acceptable. On the other hand, the AP threshold corresponds to a "neutral" situation beyond which action would be recommended. However, benchmarking based on a sample of Dutch farms (de Vries et al., 2013) revealed a threshold for identifying poor-welfare farms (worst quartile) of $6.7 \%$ of very lean cows per farm, a prevalence apparently hardly achievable in mountain farms at least before the pasture period in the present study. The potential thresholds defined for lameness/severe lameness are in contrast to the example of BCS. In fact, lame/severely lame cows were comparatively rare in the present study and the worst quartile limits ranged between $8 \%(\mathrm{BP})$ and $5 \%$ (AP). These prevalences would translate to WQ lameness scores between 60 and 80; however, these scores might be overestimated due to the difficulty in detecting mild lameness in tie-stall systems. de Vries and colleagues (2013) set the critical threshold for severely lame cows in the Netherlands at just below $12 \%$ as a result of the higher prevalence of lameness in intensive dairy farming systems compared with smallscale mountain farming systems. A different pattern is displayed for soiling of legs, for which an upper (worst) quartile level of $80 \%$ was defined when the animals were housed (BP and AP), dropping to $17 \%$ during pasture. The WQ critical threshold has been set at 50\%, resulting in a challenging target for most farmers in mountain areas during winter. Although the prevalence of some welfare outcomes (soiling, hairless patches, and very lean cows) in the worst quartile would be unacceptable according to the expert-opinion approach taken by the WQ, most prevalences in the best quartile reached $0 \%$. These results suggest that high welfare standards are widely achievable in mountain farms of the Eastern Alps.

As shown above, the definition of acceptable (expertbased) or achievable (industry-based) welfare thresholds changes according to the source consulted and the context investigated. From a pure animal welfare perspective, an expert-based approach is preferred because it provides an objective and universal judgment on acceptable (and unacceptable) farming practices. However, given the challenges and costs associated with inducing changes for welfare improvement and the time 

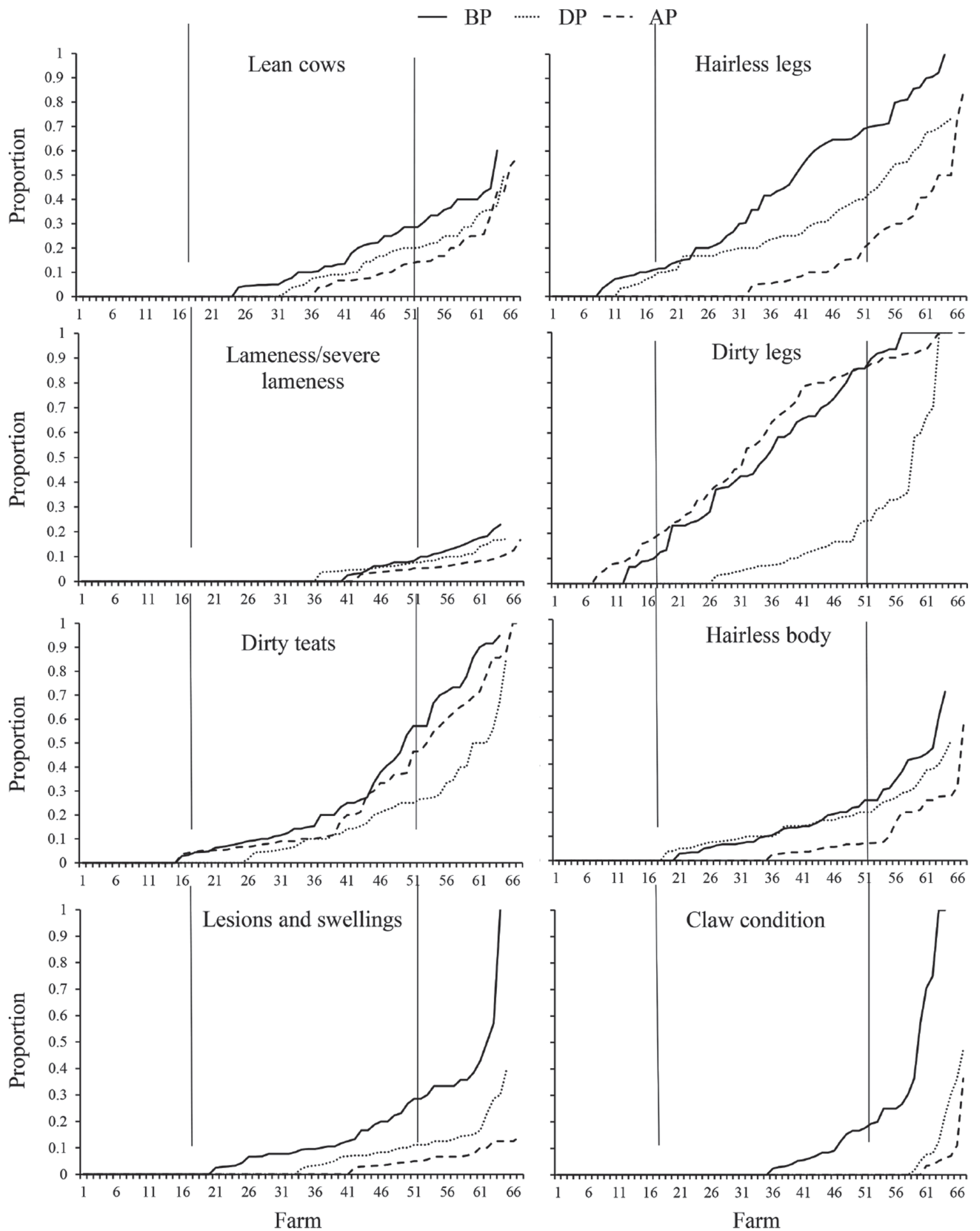

Figure 2. Distribution of each welfare condition (proportion, y-axis) in a sample of 67 small-scale dairy cattle farms (x-axis) assessed before pasture (BP), during pasture (DP), and after pasture (AP). Solid vertical lines represent thresholds at first and third quartile. 
needed to achieve significant results (Tremetsberger and Winckler, 2015), a more engaging approach should be embraced. By showing welfare outcomes that can be achieved in similar farming conditions, benchmarking seems to be more suitable than normative goals to encourage the taking of action and ultimately driving welfare improvement on farm (Main et al., 2014). The thresholds identified through benchmarking should only be used in the farming systems and contexts in which they were identified, and they should not serve as the basis for setting static minimum welfare standards. Benchmarking should instead be considered an effective on-farm welfare management strategy and a steppingstone toward continuous welfare improvement.

\section{CONCLUSIONS}

Dairy farming systems and practices vary not only between countries worldwide but also within the same country according to available resources and traditions. If the ultimate aim of welfare assessment protocols is to monitor and promote welfare improvement, this might be achieved by defining unacceptable welfare conditions as well as achievable targets from on-farm data. Although the first aspect is applicable to all farming systems, the latter should be informed by a deep understanding of different farming systems, their practices, and their challenges and can be developed over time. The present study aimed to contribute to this broader scope by providing field data from the dairy cattle transhumant system and respective animal welfare outcomes in the Eastern Alps.

\section{ACKNOWLEDGMENTS}

The authors are grateful for the economic support provided by the DairyCare COST action for Short Term Scientific Missions to Padova (Italy) and Ljubljana (Slovenia), and by the Department of Animal Science, Biotechnical Faculty of the University of Ljubljana (Slovenia). The authors also acknowledge the farmers and breeders associations for their engagement in the project.

\section{REFERENCES}

Battaglini, L., S. Bovolenta, F. Gusmeroli, S. Salvador, and E. Sturaro. 2014. Environmental sustainability of Alpine livestock farms. Ital. J. Anim. Sci. 13:431-443.

Blokhuis, H. J. 2008. International cooperation in animal welfare: The Welfare Quality ${ }^{\circledR}$ project. Acta Vet. Scand. 50(Suppl. 1):S10.

Burow, E., T. Rousing, P. T. Thomsen, N. D. Otten, and J. T. Sørensen. 2013. Effect of grazing on the cow welfare of dairy herds evaluated by a multidimensional welfare index. Animal 7:834-842.
Coignard, M., R. Guatteo, I. Veissier, A. D. des Roches, L. Mounier, A. Lehébel, and N. Bareille. 2013. Description and factors of variation of the overall health score in French dairy cattle herds using the Welfare Quality ${ }^{\circledR}$ assessment protocol. Prev. Vet. Med. 112:296-308

Corazzin, M., E. Piasentier, S. Dovier, and S. Bovolenta. 2010. Effect of summer grazing on welfare of dairy cows reared in mountain tie-stall barns. Ital. J. Anim. Sci. 9:304-312.

de Graaf, S., B. Ampe, C. Winckler, M. Radeski, L. Mounier, M. K. Kirchner, M. J. Haskell, F. J. C. M. van Eerdenburg, A. de Boyerdes Roches, S. N. Andreasen, J. Bijttebier, L. Lauwers, W. Verbeke, and F. A. M. Tuyttens. 2017. Trained-user opinion about Welfare Quality measures and integrated scoring of dairy cattle welfare. J. Dairy Sci. 100:6376-6388.

de Vries, M., E. A. Bokkers, G. van Schaik, R. Botreau, B. Engel, T. Dijkstra, and I. J. De Boer. 2013. Evaluating results of the Welfare Quality multi-criteria evaluation model for classification of dairy cattle welfare at the herd level. J. Dairy Sci. 96:6264-6273.

EFSA. 2015. Scientific opinion on the assessment of dairy cow welfare in small-scale farming systems. EFSA J. 13:4137-4239.

Fraser, D. G. 2005. Animal Welfare and the Intensification OF Animal Production: An Alternative Interpretation. Vol. 2. FAO, Rome, Italy.

Horn, M., A. Steinwidder, J. Gasteiner, L. Podstatzky, A. Haiger, and W. Zollitsch. 2013. Suitability of different dairy cow types for an Alpine organic and low-input milk production system. Livest. Sci. 153:135-146.

ISO. 2016. TS 34700: Animal welfare management-General requirements and guidance for organizations in the food supply chain. International Organization for Standardization (ISO), Geneva, Switzerland.

Keil, N. M., T. Wiederkehr, K. Friedli, and B. Wechsler. 2006. Effects of frequency and duration of outdoor exercise on the prevalence of hock lesions in tied Swiss dairy cows. Prev. Vet. Med. 74:142-153.

Leach, K. A., S. Dippel, J. Huber, S. March, C. Winckler, and H. R. Whay. 2009. Assessing lameness in cows kept in tie-stalls. J. Dairy Sci. 92:1567-1574.

Main, D. C., S. Mullan, C. Atkinson, M. Cooper, J. H. Wrathall, and H. J. Blokhuis. 2014. Best practice framework for animal welfare certification schemes. Trends Food Sci. Technol. 37:127-136.

Mattiello, S., M. Battini, E. Andreoli, and S. Barbieri. 2011. Short communication: Breed differences affecting dairy cattle welfare in traditional alpine tie-stall husbandry systems. J. Dairy Sci. 94:2403-2407.

Nash, C. G., D. Kelton, T. DeVries, E. Vasseur, J. Coe, J. Zaffino Heyeroff, V. Bouffard, D. Pellerin, J. Rushen, A. M. de Passillé, and D. Haley. 2016. Prevalence of and risk factors for hock and knee injuries on dairy cows in tiestall housing in Canada. J. Dairy Sci. 99:6494-6506.

OIE. 2015. Animal Welfare and Dairy Cattle Production System. In Terrestrial Animal Health Code. Chapter 7.11. World Organisation for Animal Health/OIE, Paris, France.

Oltenacu, P. A., and D. M. Broom. 2010. The impact of genetic selection for increased milk yield on the welfare of dairy cow. Anim. Welf. 19:39-49.

Palacio, S., R. Bergeron, S. Lachance, and E. Vasseur. 2015. The effects of providing portable shade at pasture on dairy cow behavior and physiology. J. Dairy Sci. 98:6085-6093.

Peric, T., M. Corazzin, A. Romanzin, S. Bovolenta, A. Prandi, M. Montillo, and A. Comin. 2017. Cortisol and DHEA concentrations in the hair of dairy cows managed indoor or on pasture. Livest. Sci. 202:39-43.

Piccand, V., E. Cutullic, S. Meier, F. Schori, P. Kunz, J. Roche, and P. Thomet. 2013. Production and reproduction of Fleckvieh, Brown Swiss, and 2 strains of Holstein-Friesian cows in a pasture-based, seasonal-calving dairy system. J. Dairy Sci. 96:5352-5363.

Popescu, S., C. Borda, E. A. Diugan, M. Spinu, I. S. Groza, and C. D. Sandru. 2013. Dairy cows welfare quality in tie-stall housing system with or without access to exercise. Acta Vet. Scand. 55:43-54. 
Regula, G., J. Danuser, B. Spycher, and B. Wechsler. 2004. Health and welfare of dairy cows in different husbandry systems in Switzerland. Prev. Vet. Med. 66:247-264.

Roche, J. R., J. Lee, K. Macdonald, and D. Berry. 2007. Relationships among body condition score, body weight, and milk production variables in pasture-based dairy cows. J. Dairy Sci. 90:3802-3815.

Rutherford, K. M., F. M. Langford, M. C. Jack, L. Sherwood, A. B Lawrence, and M. J. Haskell. 2009. Lameness prevalence and risk factors in organic and non-organic dairy herds in the United Kingdom. Vet. J. 180:95-105.

Sturaro, E., E. Marchiori, G. Cocca, M. Penasa, M. Ramanzin, and G. Bittante. 2013. Dairy systems in mountainous areas: Farm animal biodiversity, milk production and destination, and land use. Livest. Sci. 158:157-168.

Tremetsberger, L., and C. Winckler. 2015. Effectiveness of animal health and welfare planning in dairy herds: A review. Anim. Welf. 24:55-67.

von Keyserlingk, M. A. G., A. Barrientos, K. Ito, E. Galo, and D. M. Weary. 2012. Benchmarking cow comfort on North American freestall dairies: Lameness, leg injuries, lying time, facility design, and management for high-producing Holstein dairy cows. J. Dairy Sci. 95:7399-7408.

Welfare Quality. 2009. Welfare Quality assessment protocol for cattle. WQ Consortium, Lelystad, the Netherlands.

Whay, H. R., D. Main, L. Green, and A. Webster. 2003. Assessment of the welfare of dairy caftle using animal-based measurements: direct observations and investigation of farm records. Vet. Rec. 153:197-202.

Wymann von Dach, S., R. Romeo, A. Vita, M. Wurzinger, and T. Kohler. 2013. Mountain farming is family farming: A contribution from mountain areas to the International Year of Family Farming 2014. FAO, Rome, Italy.

Zuliani, A., A. Romanzin, M. Corazzin, S. Salvador, J. Abrahantes, and S. Bovolenta. 2017. Welfare assessment in traditional mountain dairy farms: Above and beyond resource-based measures. Anim. Welf. 26:203-211. 